\title{
Airport Service Vehicle Scheduling
}

Kenneth Kuhn Univ. of Canterbury (formerly NASA)
Steffen Loth

DLR 

Why Service Vehicle Scheduling

"For most airports there is a dominance of delays due to gate congestion" (Idris et al., ATM 1998)

Interdependence of gates, airports

Very little service vehicle research to date, none from the perspective of a service provider

Available research has focused on describing the turnaround process 



\section{The CARMA Project}
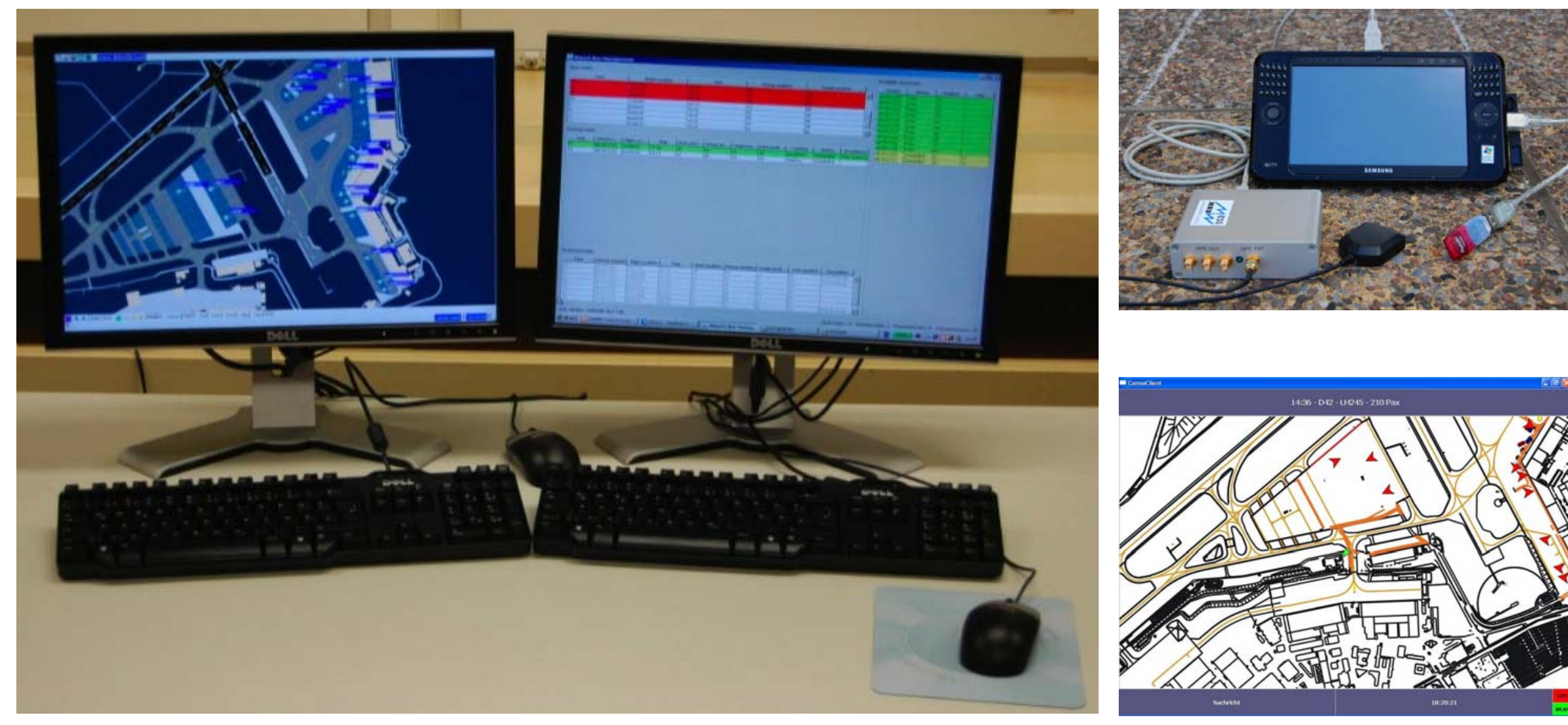

Hámburg Airport

Niedersächsisches Ministerium für Wirtschaft, Arbeit und Verkehr

\section{AIRXSYS}

UH

\section{(5)AIRBUS} TECHNISCHE UNIVERSITÄT A.9. ZU BRAUNSCHWEIG
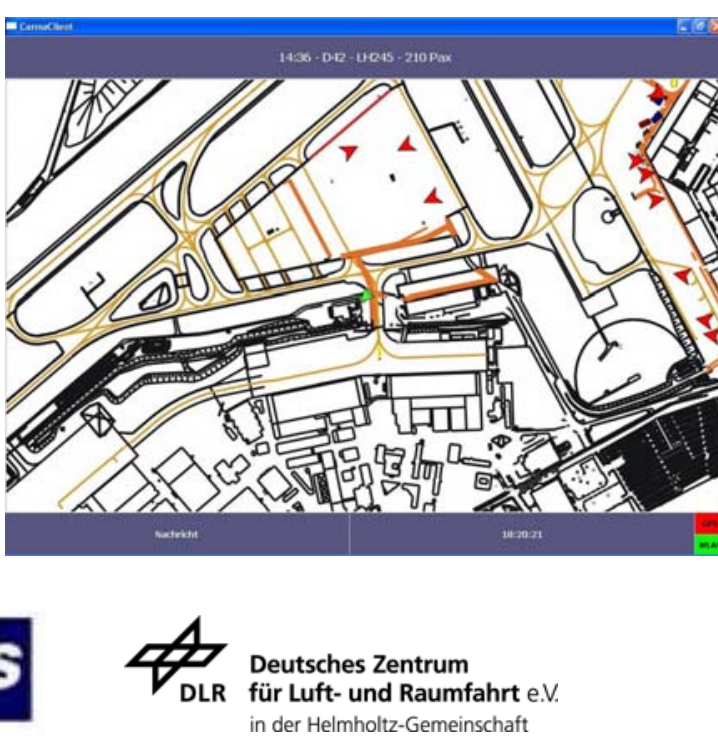

in der Helmholtz-Gemeinschaft 


\section{Scheduling Algorithms}

Decisions

assign service vehicles aircraft to service assign times when service is to begin

Objectives

minimize delay aircraft absorb minimize distance service vehicles travel minimize number of service vehicles required

Difficulties

aircraft assignment has exponential possibilities aircraft sequencing has factorial possibilities 


\section{Scheduling Algorithms}

Current

periodically see if aircraft is about to require service use vehicles that have been idle the longest

Greedy

use vehicles that are closest to aircraft

Moving time window

periodically solve static scheduling problem assign service vehicles according to results 


\section{Scheduling Algorithms: Moving Time Window}

Planning horizon need not equal assignment horizon (examine schedule over next hour, every ten minutes)

Assignment horizon should depend on extent of uncertainty

Planning horizon should depend on computational power

In cases where planning horizon is unreasonably short, test heuristic approaches like genetic algorithms

"Clever optimization algorithms are best" 


\section{Scheduling Algorithms: Clever Optimization}

Modify constraints to discourage fractional variables

Constraints on service times sum across binary sequencing variables

$$
\begin{array}{cc}
b_{j} \geq\left(F_{x}+\frac{D_{x, j}}{V}\right) a_{0, j}^{x} & \forall j \in I, x \in X \\
\text { becomes } & \forall j \in I \\
b_{j} \geq \sum_{x \in X}\left[\left(F_{x}+\frac{D_{x, j}}{V}\right) a_{0, j}^{x}+\sum_{i \in I}\left(T_{i}+S_{i}+\frac{D_{i, j}}{V}\right) a_{i, j}^{x}\right] & \forall j \in
\end{array}
$$

Add constraints to penalize cyclic flow

$$
\sum_{x \in X}\left(a_{i, j}^{x}+a_{j, i}^{x}\right) \leq 1 \quad \forall i, j \in I
$$




\section{Scheduling Algorithms: Clever Optimization}

Branch based on vehicle assignment and task sequencing, never on individual variables

(Somewhat) more detailed explanation in paper

Methods applicable to other vehicle routing problems, including arrival scheduling 


\section{Scheduling Algorithms: Genetic Algorithm}

Technique borrowed from arrival scheduling assign aircraft to runways / vehicles sequence aircraft schedule based on sequence (trivial)

\begin{tabular}{l|l|l|l|l|}
\cline { 2 - 5 } Aircraft & 1 & 2 & 3 & 4 \\
\hline Individual & $\mathbf{0 . 4 5}$ & $\mathbf{1 . 6 3}$ & $\mathbf{1 . 4 8}$ & $\mathbf{1 . 3 1}$ \\
\hline $\begin{array}{l}\text { Vehicle } \\
\text { Assignment }\end{array}$ & Vehicle 1 & Veh 2 & Veh 2 & Veh 2 \\
\hline $\begin{array}{l}\text { Ordering } \\
\text { Instructions }\end{array}$ & 1st Task & 3rd & 2nd & 1st \\
\cline { 2 - 5 } & \multicolumn{2}{|l}{}
\end{tabular}




\section{Simulation Studies: HAM}

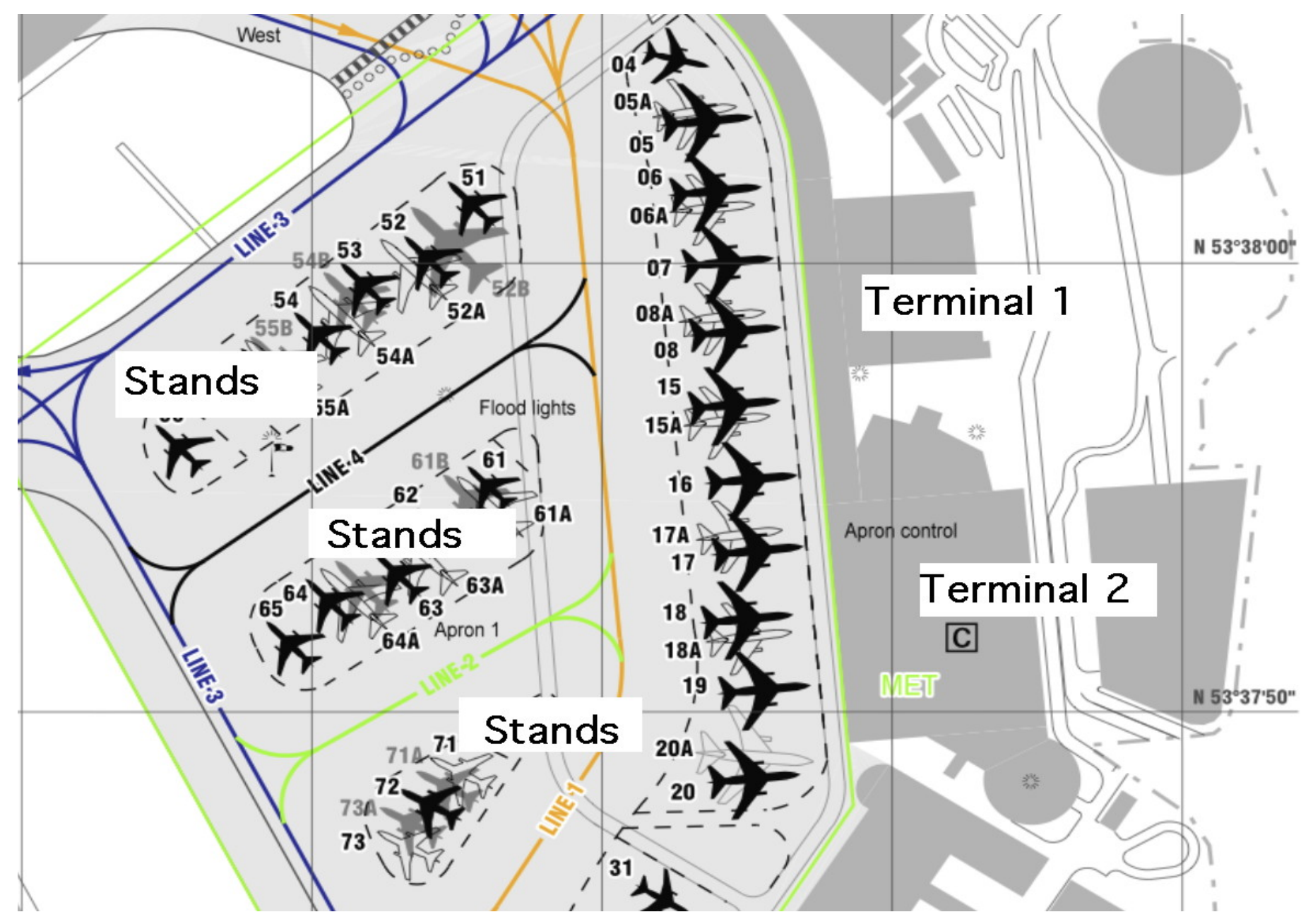




\section{Simulation Studies: HAM}

200 scenarios given to various scheduling algorithms

17 aircraft requesting service from 6 service vehicles in each scenario (a busy hour or two at HAM)

Over $10^{13}$ ways to assign aircraft

For each assignment, as many as $10^{14}$ ways to sequence tasks 




\section{Simulation Studies: DFW}

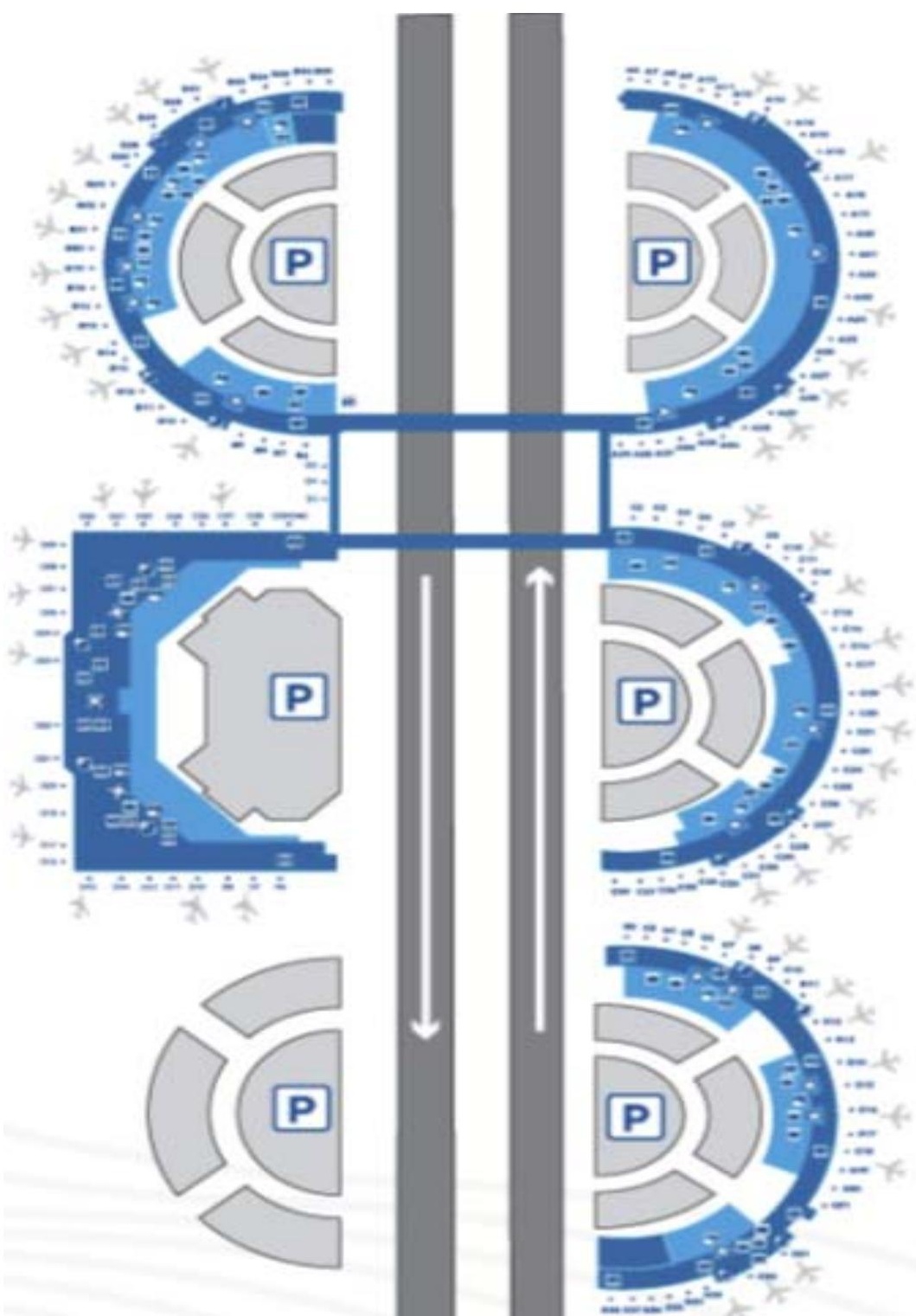







\section{Conclusion}

Vehicle management systems have significant potential at both small and large airports

reducing delay aircraft absorb

reducing distance service vehicles travel

reducing service vehicle fleet size

Delay aircraft absorb waiting for service vehicles also a function of arrival and departure time distributions

Established clever optimization and genetic algorithms for scheduling 